\title{
Highly polarized electrically driven single-photon emission from a non-polar InGaN quantum dot
}

C. C. Kocher, T. J. Puchtler, J. C. Jarman, T. Zhu, T. Wang, L. Nuttall, R. A. Oliver, and R. A. Taylor

Citation: Appl. Phys. Lett. 111, 251108 (2017); doi: 10.1063/1.5008720

View online: https://doi.org/10.1063/1.5008720

View Table of Contents: http://aip.scitation.org/toc/apl/111/25

Published by the American Institute of Physics

\section{Articles you may be interested in}

Burying non-radiative defects in InGaN underlayer to increase InGaN/GaN quantum well efficiency Applied Physics Letters 111, 262101 (2017); 10.1063/1.5007616

Temperature-dependent fine structure splitting in InGaN quantum dots

Applied Physics Letters 111, 053101 (2017); 10.1063/1.4996861

UV detector based on InAIN/GaN-on-Si HEMT stack with photo-to-dark current ratio $>10^{7}$ Applied Physics Letters 111, 251103 (2017); 10.1063/1.5004024

Electrically driven quantum light emission in electromechanically tuneable photonic crystal cavities Applied Physics Letters 111, 251101 (2017); 10.1063/1.5008590

Epitaxy of GaN in high aspect ratio nanoscale holes over silicon substrate Applied Physics Letters 111, 252101 (2017); 10.1063/1.5002529

Multi-excitonic emission from Stranski-Krastanov GaN/AIN quantum dots inside a nanoscale tip Applied Physics Letters 111, 243102 (2017); 10.1063/1.5004417

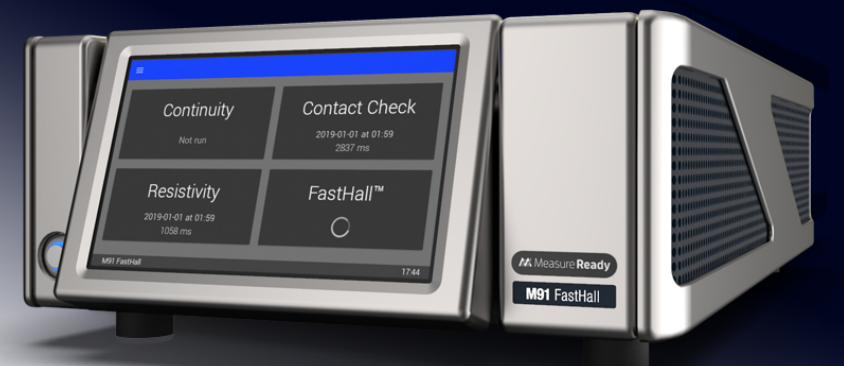

\section{MNM Measure Ready M91 FastHall ${ }^{\mathrm{TM}}$ Controller \\ A revolutionary new instrument for complete Hall analysis}




\title{
Highly polarized electrically driven single-photon emission from a non-polar InGaN quantum dot
}

\author{
C. C. Kocher, ${ }^{1, a)}$ T. J. Puchtler, ${ }^{1}$ J. C. Jarman, ${ }^{2}$ T. Zhu, ${ }^{2}$ T. Wang, ${ }^{1}$ L. Nuttall, ${ }^{1}$ R. A. Oliver, ${ }^{2}$ \\ and R. A. Taylor ${ }^{1}$ \\ ${ }^{1}$ Department of Physics, University of Oxford, Parks Road, Oxford OX1 3PU, United Kingdom \\ ${ }^{2}$ Department Materials Science and Metallurgy, University of Cambridge, 27 Charles Babbage Road, \\ Cambridge CB3 OFS, United Kingdom
}

(Received 9 October 2017; accepted 4 December 2017; published online 20 December 2017)

\begin{abstract}
Nitride quantum dots are well suited for the deterministic generation of single photons at high temperatures. However, this material system faces the challenge of large in-built fields, decreasing the oscillator strength and possible emission rates considerably. One solution is to grow quantum dots on a non-polar plane; this gives the additional advantage of strongly polarized emission along one crystal direction. This is highly desirable for future device applications, as is electrical excitation. Here, we report on electroluminescence from non-polar $\mathrm{InGaN}$ quantum dots. The emission from one of these quantum dots is studied in detail and found to be highly polarized with a degree of polarization of 0.94 . Single-photon emission is achieved under excitation with a constant current giving a $g^{(2)}(0)$ correlation value of 0.18 . The quantum dot electroluminescence persists up to temperatures as high as $130 \mathrm{~K}$. Published by AIP Publishing. https://doi.org/10.1063/1.5008720
\end{abstract}

Single-photon sources (SPSs) have great potential in the fields of quantum cryptography ${ }^{1}$ and quantum computation. ${ }^{2}$ Semiconductor quantum dots (QDs) are well suited to these applications as they allow for deterministic single-photon emission. In particular, nitride QDs show promise because of their large band offsets and exciton binding energies, enabling operation at room temperature ${ }^{3,4}$ and above. ${ }^{5}$ Furthermore, the emission energies of InGaN QDs can be adapted via ternary alloy mixing, spanning between the near infrared region with $\mathrm{InN}\left(0.7^{6}-0.9 \mathrm{eV}^{7}\right)$ and the near $\mathrm{UV}$ range with $\mathrm{GaN}\left(3.4 \mathrm{eV}^{8}\right)$. Operation in the blue wavelength range is particularly desirable to match the maximum efficiency of single-photon detectors. ${ }^{9}$ Blue single-photon sources operating at room temperature have potential for secure short range communication on earth or satellite-to-satellite communication.

A major step towards practical applications is represented by electrical injection rather than optical excitation. This enables lower costs to be achieved and a much more robust and compact setup. Only two groups have reported on electrically driven InGaN QDs emitting blue single photons, achieved by means of a restricted quantum well (QW) acting as a QD. ${ }^{10-12}$ These were either grown in a nanowire ${ }^{10,11}$ or as a planar QW and then etched down to pillars. ${ }^{12}$ In contrast, we grow selfassembled QDs on a planar surface, allowing the application of conventional process flows to the device fabrication and thereby enabling future development of more complex device structures such as photonic crystal cavities.

Nitride materials are peculiar in that they feature large spontaneous and piezo-electric fields along the $c$-direction, resulting in significant electric fields across heterostructure boundaries. This leads to the separation of the electron and hole wavefunctions of an exciton, resulting in longer lifetimes and decreased recombination rates. A workaround for

a) claudius.kocher@physics.ox.ac.uk this problem is growth on a non-polar plane perpendicular to the $c$-plane, which has been shown to reduce excitonic lifetimes by one order of magnitude. ${ }^{13,14}$ Furthermore, an additional advantage is the highly polarized emission along one crystal axis. ${ }^{15}$ This is due to the anisotropic strain in the growth plane, which separates the upper two valence bands. ${ }^{16}$ Polarized emission along a fixed direction is highly desirable as quantum cryptography schemes rely on polarized SPSs. ${ }^{1}$ Here, we demonstrate highly polarized singlephoton emission from an electrically driven InGaN QD.

The $p-i-n$ LED structure was grown by metal-organic vapour phase epitaxy on a non-polar (1120) $a$-plane $\mathrm{GaN}$ pseudo-substrate, where InGaN QDs were embedded in the intrinsic GaN layer. Electrical devices were subsequently patterned using UV photolithography and fabricated by chlorine based inductively coupled plasma etching. The structure is shown in the left inset of Fig. 1. The InGaN QDs were grown using a quasi-two-temperature method, ${ }^{17}$ in a mode similar to the Stranski-Krastanov mode. To increase the QD density, 5 QD layers were grown on top of each other with

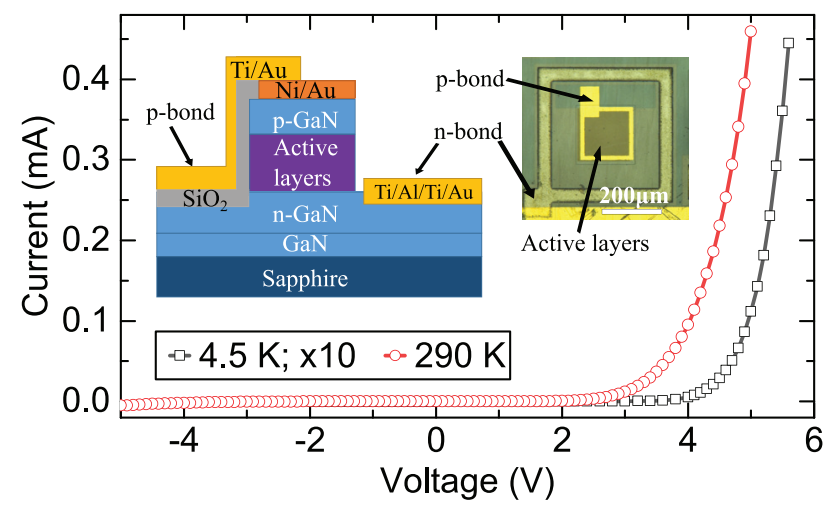

FIG. 1. IV-curves at room temperature and $4.5 \mathrm{~K}$ show diode-like behavior of the device. Left inset: LED layer schematic. Right inset: Microscopy image of the studied LED. 
$7.5 \mathrm{~nm}$ GaN separating them. They were sandwiched between layers of $2 \mu \mathrm{m}$ thick $n$-GaN on the bottom and $130 \mathrm{~nm}$ thick $p$-GaN on the top. For $n$-GaN, the Si dopant concentration was $3 \times 10^{18} \mathrm{~cm}^{-3}$ and the $\mathrm{Mg}$ dopant concentration for $p$-GaN was $3 \times 10^{19} \mathrm{~cm}^{-3} \cdot n$-GaN was contacted using a layer sequence of $\mathrm{Ti} / \mathrm{Al} / \mathrm{Ti} / \mathrm{Au}$ with the thickness of $15 / 10 / 5 / 80 \mathrm{~nm}$. $p$-GaN was contacted with transparent layers of $\mathrm{Ni} / \mathrm{Au} 4.5 \mathrm{~nm}$ thick, to allow for improved current spreading, and layers of Ti/Au with thicknesses of 20 and $80 \mathrm{~nm}$ were then added. A $200 \mathrm{~nm}$ thick layer of $\mathrm{SiO}_{2}$ is used to improve the mechanical stability of the $p$-GaN contact. The active area of the device is $200 \times 200 \mu \mathrm{m}^{2}$. An optical microscopy image of the studied LED is shown in the right inset of Fig. 1.

Micro-electroluminescence ( $\mu$-EL) measurements were performed using a confocal microscope. The sample was held at $4.5 \mathrm{~K}$ in a closed-cycle helium cryostat (AttoDRY800) with a nanopositioning system, including a heater to access temperatures up to $300 \mathrm{~K}$. The sample was excited electrically using a DC-voltage supplied by a source-measure unit (Keithley 236). $\mu$-EL was collected with a $100 \times$ objective with a numerical aperture of 0.5 . It was imaged onto a multimode fiber with a core diameter of $25 \mu \mathrm{m}$, corresponding to a $1 \mu \mathrm{m}$-spot on the sample. The fiber end was connected to either a spectrometer or a Hanbury-Brown-Twiss setup (HBT). A $0.5 \mathrm{~m}$ spectrometer (Shamrock 500i) dispersed $\mu$ EL with a $12001 / \mathrm{mm}$ grating onto a Peltier cooled Si-based charge-coupled device, yielding a pixel resolution of $0.04 \mathrm{~nm}$. The HBT setup was used to determine the second-order correlation function of the QD emission. $\mu$-EL was spectrally isolated using two tunable bandpass filters and sent through a non-polarizing 50:50 beam-splitter to a pair of photomultiplier tubes (PMTs, Hamamatsu H10720) mounted on the two exit faces. The signals from the two PMTs were time-tagged by a time-correlated single-photon module (Picoquant TimeHarp 260) with a channel resolution of 25 ps. From there, a histogram was built up over the time delay between two detection events, each on one of the PMTs. The polarization properties of $\mu$-EL were probed by a rotatable half-wave plate and a fixed polarizer located between the objective and the fiber. The combination of a half-wave plate and a polarizer was used to minimize the spatial walkoff of the QD $\mu$-EL.

Initial measurements involved taking IV-curves at room temperature and at $4.5 \mathrm{~K}$, shown in Fig. 1. They show the expected diode behavior with a flat response under reverse bias and low forward bias, with voltage thresholds of 3.9(1) and $4.8(1) \mathrm{V}$, respectively, followed by an exponential increase. The commonly observed larger onset voltage at lower temperatures is due to a reduction in hole mobility and difficulty in activating donors. ${ }^{18,19}$

When applying voltages larger than $4 \mathrm{~V}$ at $4.5 \mathrm{~K}$, we observe $\mu$-EL with two spectral contributions: sharp peaks between $410 \mathrm{~nm}$ and $550 \mathrm{~nm}$ on a broad background of the same range centered at $500 \mathrm{~nm}$. Because the linewidths of the peaks range from a few $\mathrm{meV}$ down to the resolution limit, we attribute these to QDs, whereas the broad background should originate from the underlying QW acting as a wetting layer. The spectrum of the QD under question is shown in Fig. 2, featuring a $1.2 \mathrm{meV}$-wide peak at $420 \mathrm{~nm}$ on

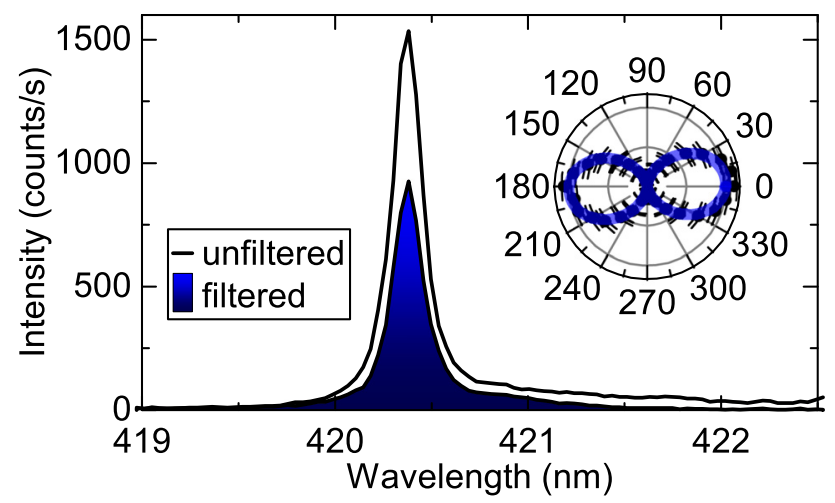

FIG. 2. Spectrum of the studied QD at a driving voltage of $5.8 \mathrm{~V}$. The shaded spectrum shows the same QD but filtered for the HBT measurement. Inset: Integrated QD intensity as a function of the polarizer angle. The fit according to Malus's law gives a DOLP of 0.94(1). The polarization angle $\theta_{0}$ is perpendicular to the $c$-direction within $10^{\circ}$.

a low, but discernible background. Its polarization degree was probed in the following way: spectra were taken for $5 \mathrm{~s}$ every $10^{\circ}$ over a range of $360^{\circ}$ and fitted with a Gaussian function. This line shape is found to be closest to the actual QD line shape, for which the (Gaussian) inhomogeneous part dominates over the (Lorentzian) homogeneous part. ${ }^{20}$ Phonons are believed to play only a minor role at $4.5 \mathrm{~K}^{21}$ The integrated intensity obtained is plotted as a function of the polarization angle in the inset of Fig. 2. It is evident that $\mu$-EL from the QD is strongly linearly polarized. Here, an angle of $13(3)^{\circ}$ corresponds to the crystallographic $m$-axis, which is determined relative to surface striations along the $c$-direction ${ }^{22}$ observed in optical microscopy images (see the right inset of Fig. 1). The polarization angle of the QD and its degree of linear polarization (DOLP) are determined by fitting the integrated intensity with Malus's Law $I(\theta)=I_{\max } \cos ^{2}\left(\theta-\theta_{0}\right)$ $+I_{\min } \sin ^{2}\left(\theta-\theta_{0}\right)$ and calculating the polarization degree as $\mathrm{DOLP}=\left(I_{\max }-I_{\min }\right) /\left(I_{\max }+I_{\min }\right)=0.94(1)$. This high value is expected as a photoluminescence (PL) study on InGaN QDs grown on the $a$-plane showed a high average degree of linear polarization. ${ }^{23}$ Interestingly, this polarization degree is significantly higher than the degree of 0.7 for previously reported QD $\mu$-EL. ${ }^{10,12}$ Furthermore, in contrast to previous reports of polarized emission from InGaN QDs, ${ }^{10,12,24}$ the polarization mechanism does not require complicated and laborious processing, but it is inherent in the growth method due to the anisotropic strain profile in the growth plane. ${ }^{16}$ The polarization angle $\theta_{0}$ of this QD lies within $10^{\circ}$ of the $m$-direction. Alignment to a crystal direction has been shown previously for $a$-plane InGaN QDs in PL studies. ${ }^{15,23}$ Additional $\mu$-EL results regarding the distributions of polarization degrees and angles of QDs will be reported elsewhere.

The emission statistics of the studied QD were probed under constant current excitation. The normalized secondorder correlation function $g^{(2)}(\tau)=\langle I(t) I(t+\tau)\rangle /\langle I(t)\rangle\langle I(t$ $+\tau)\rangle$ is measured without the use of any position optimisation. The QD spectrum filtered for the HBT measurement is displayed in Fig. 2 for a driving voltage of $5.8 \mathrm{~V}$. The HBT trace obtained is shown in Fig. 3. The mean coincidence per time bin was 467 . The dip at zero delay is evident, being $0.66(4)$ as extracted with a fit of the form 


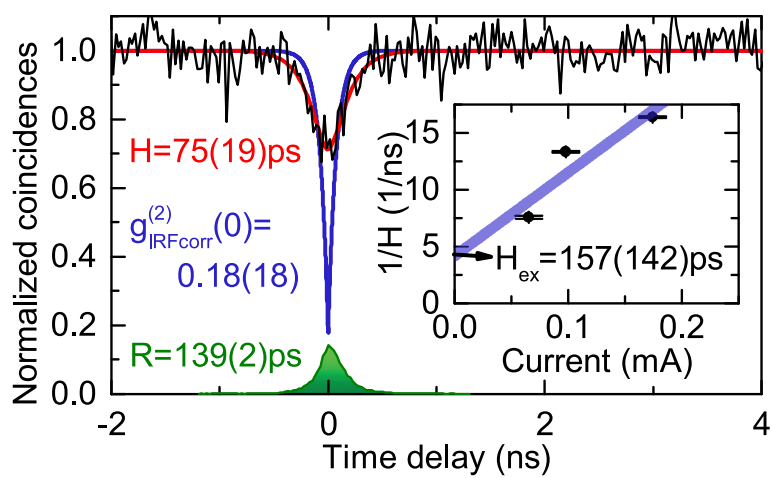

FIG. 3. HBT measurement at a driving voltage of $5.8 \mathrm{~V}$. The black line shows the measured HBT trace. The fit (red) is the convolution of the second-order correlation function [Eq. (1)] with the IRF [Eq. (2)]. The IRFcorrected HBT trace (blue) dips down to $g_{\mathrm{IRF} \text { ( }}^{(2)}(0)=0.18(18)$. Below, the IRF (green) is shown with its mean decay time $R$. Inset: Plot of the HBT time constants $H$ as a function of the injection current. The exciton lifetime $H_{\text {ex }}$ is obtained from the ordinate axis offset of the linear regression.

$$
g^{(2)}(\tau)=1-A \times \exp (-|\tau| / H)
$$

which assumes a single exponential decay of the HBT trace with a time constant $H$.

However, previous PL studies on $a$-plane QDs show an exciton lifetime around $500 \mathrm{ps},{ }^{13}$ which is shorter than in previous reports on DC-driven $\mu$-EL HBT traces of polar QDs (up to several nanoseconds). ${ }^{12}$ These lifetimes approach the time constant of the instrument response function (IRF) of the HBT setup. Therefore, here we have to take the IRF into account in order to extract the real $g^{(2)}(0)$ properly. The IRF was measured with a $400 \mathrm{~nm}$, pulsed femtosecond laser source and is shown in Fig. 3. It is fitted well by a doublesided exponential decay

$$
\operatorname{IRF}(\tau)=B \times \exp (-|\tau| / R)
$$

with a decay time of $R=139(2) \mathrm{ps}$. The value and uncertainty were determined from the mean and standard deviation of 48 independent measurements. Thus, the shape of the measured HBT trace is a convolution of the second-order correlation function [Eq. (1)] and the IRF [Eq. (2)]. The obtained analytical expression is

$$
g_{\text {conv }}^{(2)}(\tau)=1-\frac{A \times H}{R^{2}-H^{2}} \times\left(R \mathrm{e}^{-|\tau| / R}-H \mathrm{e}^{-|\tau| / H}\right) .
$$

The data were fitted with Eq. (3) with the IRF decay time $R$ fixed to the above value, as shown in red in Fig. 3. It yields the IRF-corrected $g_{\text {IRFcorr }}^{(2)}(\tau)$ as shown in blue, dipping down to $g_{\text {IRFcorr }}^{(2)}(0)=0.18(0.18)$. This demonstrates that we have electrically driven single-photon emission. It should be noted that the large uncertainty is due to the long IRF decay time and the finite channel bin width of $25 \mathrm{ps}$. The remaining $g_{\text {IRFcorr }}^{(2)}(0)$ may well result from the background. However, knowledge of the exact QD line shape is crucial for a background correction. Here, this is not directly measurable due to the impact of spectral diffusion and phonon scattering. This limits the value of a background correction. However, it is certain that $g_{\text {IRFcorr }}^{(2)}(0)$ would be lower without QW emission. Future experiments will explore methods of reducing the background. With regard to the limited HBT timing resolution, future experiments will use pulsed electrical excitation to circumvent it.

Along with the dip depth, the fit provides the HBT time constant $H$ of the QD. This measure is composed of the exciton lifetime $H_{\mathrm{ex}}$ and the electrical pump rate $p$ in the following way: ${ }^{25} 1 / H=1 / H_{\mathrm{ex}}+p$. In order to extract the exciton lifetime, an HBT trace was taken for two additional, different drive voltages (5.6 and $6.1 \mathrm{~V}$ ) and fitted as previously discussed. The mean coincidence counts per time bin were 524 and 671 for the lower and higher currents, respectively. The data are plotted as a function of the injection current, which is proportional to the pump rate, in the inset of Fig. 3. A linear fit gives a lifetime of $H_{\mathrm{ex}}=157(142) \mathrm{ps}$, which is well within the range of our measurements on similar samples.

Of course, one might consider the possibility that the single-photon emission does not stem from a QD, but an exciton bound to a defect or an impurity. Defect related emission would show a discontinuous wavelength shift with temperature, with the bound excitons thermalizing to free excitons, yielding two distinct peaks at a certain temperature. $^{26}$ To differentiate between these two possibilities, a series of spectra were taken at a constant voltage of $6.7 \mathrm{~V}$ for different temperatures, shown in Fig. 4(a). One can see the shift to lower energy, broadening and intensity decrease with increasing temperature, which will be examined in further detail. Each spectrum is fitted with a Gaussian function as a reasonable line shape approximation to extract the relevant peak parameters. However, one should note that these depend on the injection voltage and current, of which only the latter could be fixed. This especially affects the intensity and the linewidth. However, the temperature shift of the QD peak energy is much larger, as shown in Fig. 4(b). Thus, it can be approximated with a commonly used model. The QD peak shifts towards lower energies as predicted for semiconductors by the Varshni equation. ${ }^{27}$ The data are very well

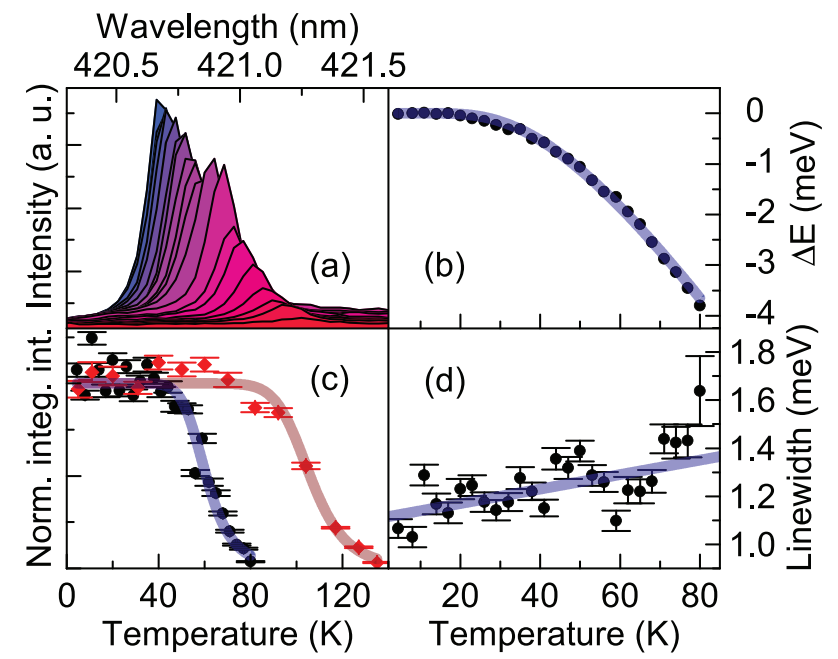

FIG. 4. Temperature dependent studies. (a) Spectra between $4.5 \mathrm{~K}$ and $80 \mathrm{~K}$ at a driving voltage of 6.7 V. (b) QD peak energy shift against temperature. Uncertainties are smaller than the data points. The fit is an improved Varshni model specified in the text. (c) Integrated QD intensity dependence against temperature for the QD used for the HBT measurement in Fig. 3 (black circles) and a second QD (red squares, driving voltage 6.2 V). The lines are a guide to the eye. (d) QD linewidth against the temperature. The line is a guide to the eye. 
fitted by an improved model $E(T)=E_{0}-S\langle\hbar \omega\rangle[\operatorname{coth}(\langle\hbar \omega\rangle /$ $2 k T)-1]$ by O'Donnell and Chen. ${ }^{28}$ Here, $E_{0}$ represents the emission energy at near $0 \mathrm{~K},\langle\hbar \omega\rangle=12.2(4) \mathrm{meV}$ is an average phonon energy, and $S=0.73(4)$ is a dimensionless coupling parameter. A continuous energy shift is observed for the temperature dependence, confirming that the emission originates from a QD. The integrated intensity is plotted in Fig. 4(c), normalized to its maximum. The intensity stays constant up to $50 \mathrm{~K}$ and then decreases. This is fitted well by a single channel Arrhenius-type model $I(T)=I_{0} /[1+A$ $\times \exp (-E / k T)]$, which just serves as a guide to the eye as explained earlier. Additionally, the behavior of a second QD is shown. Its integrated intensity only begins to decrease at $80 \mathrm{~K}$, and it lasts up to $130 \mathrm{~K}$. It would be thus suitable for operation above the temperature of liquid nitrogen. The potential of non-polar InGaN QDs for even higher temperature operation was recently shown ${ }^{29}$ in PL studies with emission up to $250 \mathrm{~K}$, including polarized single-photon emission up to $220 \mathrm{~K}$. Here, however, the absolute intensity of the second QD is too low to record an HBT trace. In general, the intensity of our sample is limited by the low extraction efficiency. By patterning our sample into pillars, ${ }^{29}$ we foresee room for improvement in three areas: increased extraction efficiency due to the guiding effect of the pillars, reduction of background emission due to the pillar diameter being much smaller than the optical diffraction limit, and better temperature performance due to a change in the phononic structure in the pillars. In Fig. 4(d), the linewidth of the QD used for the HBT measurement is shown. It increases linearly and slowly with temperature. The linewidth at low temperatures is $\sim 1 \mathrm{meV}$, which is well above the Fourier limit, determined by the short lifetime. The low temperature linewidth is hence very likely caused by spectral diffusion. ${ }^{30}$

To conclude, micro-electroluminescence measurements were performed on a non-polar InGaN QD in a planar LED structure. Evidence for highly polarized single-photon emission was presented. The dip of the second order correlation function was as low as $g^{(2)}(0)=0.18(18)$ after correction for the instrument response function. Furthermore, a lifetime of 156(142) ps was extracted, showing the ultrafast emission of the non-polar QD. Finally, temperature-dependent studies underpinned the assignment of the emission as originating from a QD. QD micro-electroluminescence was shown to persist up to $130 \mathrm{~K}$.

Data Availability: All data presented are available free of charge at the DOI: http://doi.org/10.5287/bodleian: Ae1RGxd4A.

This work was supported by the EPSRC (EP/M012379/ 1 and EP/M011682/1). C.C.K. is grateful for the support provided by a Clarendon Scholarship, a Mary Frances and
Philip Wagley Graduate Scholarship, and EPSRC funding. T.W. is grateful for the award of a National Science Scholarship (NSS) as Ph.D. funding by the Singapore Agency for Science, Technology and Research (A*STAR). R.A.O. is grateful to the Leverhulme Trust and the Royal Academy of Engineering for a Senior Research Fellowship.

${ }^{1}$ C. H. Bennett and G. Brassard, in Proceedings of IEEE International Conference on Computers, Systems and Signal Processing (1984), p. 175.

${ }^{2}$ E. Knill, R. Laflamme, and G. J. Milburn, Nature 409, 46 (2001).

${ }^{3}$ S. Deshpande, T. Frost, A. Hazari, and P. Bhattacharya, Appl. Phys. Lett. $\mathbf{1 0 5}, 141109$ (2014).

${ }^{4}$ M. J. Holmes, K. Choi, S. Kako, M. Arita, and Y. Arakawa, Nano Lett. 14, 982 (2014).

${ }^{5}$ M. J. Holmes, S. Kako, K. Choi, M. Arita, and Y. Arakawa, ACS Photonics 3, 543 (2016)

${ }^{6}$ V. Davydov, A. Klochikhin, R. Seisyan, V. Emtsev, S. Ivanov, F. Bechstedt, J. Furthmüller, H. Harima, A. Mudryi, J. Aderhold, O. Semchinova, and J. Graul, Phys. Status Solidi (B) 229, r1 (2002).

${ }^{7}$ S. Z. Wang, S. F. Yoon, Y. X. Xia, and S. W. Xie, J. Appl. Phys. 95, 7998 (2004).

${ }^{8}$ H. P. Maruska and J. J. Tietjen, Appl. Phys. Lett. 15, 327 (1969).

${ }^{9}$ R. H. Hadfield, Nat. Photonics 3, 696 (2009).

${ }^{10}$ S. Deshpande, J. Heo, A. Das, and P. Bhattacharya, Nat. Commun. 4, 1675 (2013).

${ }^{11}$ S. Deshpande and P. Bhattacharya, Appl. Phys. Lett. 103, 241117 (2013).

${ }^{12}$ L. Zhang, C. H. Teng, P. C. Ku, and H. Deng, Appl. Phys. Lett. 108, 153102 (2016).

${ }^{13}$ B. P. L. Reid, T. Zhu, C. Kocher, F. Oehler, R. Emery, M. J. Kappers, R. A. Oliver, and R. A. Taylor, Phys. Status Solidi C 11, 702 (2014).

${ }^{14}$ T. J. Puchtler, T. Wang, C. X. Ren, F. Tang, R. A. Oliver, R. A. Taylor, and T. Zhu, Nano Lett. 16, 7779 (2016).

${ }^{15}$ B. P. L. Reid, C. Kocher, T. Zhu, F. Oehler, C. C. S. Chan, R. A. Oliver, and R. A. Taylor, Appl. Phys. Lett. 106, 171108 (2015).

${ }^{16}$ S. Schulz, T. J. Badcock, M. A. Moram, P. Dawson, M. J. Kappers, C. J. Humphreys, and E. P. O'Reilly, Phys. Rev. B 82, 125318 (2010).

${ }^{17}$ T. Wang, T. J. Puchtler, T. Zhu, J. C. Jarman, R. A. Oliver, and R. A. Taylor, Phys. Status Solidi B 254, 1600724 (2017).

${ }^{18}$ C. H. Wang, J. R. Chen, C. H. Chiu, H. C. Kuo, Y. L. Li, T. C. Lu, and S. C. Wang, IEEE Photonics Technol. Lett. 22, 236 (2010).

${ }^{19}$ P. Perlin, M. Osiński, P. G. Eliseev, V. A. Smagley, J. Mu, M. Banas, and P. Sartori, Appl. Phys. Lett. 69, 1680 (1996).

${ }^{20}$ M. Holmes, S. Kako, K. Choi, M. Arita, and Y. Arakawa, Phys. Rev. B 92, 115447 (2015).

${ }^{21}$ F. Rol, S. Founta, H. Mariette, B. Daudin, L. S. Dang, J. Bleuse, D. Peyrade, J.-M. Gérard, and B. Gayral, Phys. Rev. B 75, 125306 (2007).

${ }^{22}$ T. Zhu, F. Oehler, B. P. L. Reid, R. M. Emery, R. A. Taylor, M. J. Kappers, and R. A. Oliver, Appl. Phys. Lett. 102, 251905 (2013).

${ }^{23}$ T. Wang, T. J. Puchtler, S. K. Patra, T. Zhu, M. Ali, T. J. Badcock, T. Ding, R. A. Oliver, S. Schulz, and R. A. Taylor, Nanophotonics 6(5), 1175 (2017).

${ }^{24}$ C. H. Teng, L. Zhang, T. A. Hill, B. Demory, H. Deng, and P. C. Ku, Appl. Phys. Lett. 107, 191105 (2015).

${ }^{25}$ P. Michler, A. Imamoglu, M. D. Mason, P. J. Carson, G. F. Strouse, and S. K. Buratto, Nature 406, 968 (2000).

${ }^{26}$ M. Belloeil, B. Gayral, and B. Daudin, Nano Lett. 16, 960 (2016).

${ }^{27}$ Y. Varshni, Physica 34, 149 (1967).

${ }^{28}$ K. P. O’Donnell and X. Chen, Appl. Phys. Lett. 58, 2924 (1991).

${ }^{29}$ T. Wang, T. J. Puchtler, T. Zhu, J. C. Jarman, L. P. Nuttall, R. A. Oliver, and R. A. Taylor, Nanoscale 9, 9421 (2017).

${ }^{30}$ B. P. L. Reid, T. Zhu, T. J. Puchtler, L. J. Fletcher, C. C. S. Chan, R. A. Oliver, and R. A. Taylor, Jpn. J. Appl. Phys., 52, 08 JE01 (2013). 